\title{
Laparoscopic Treatment of Gastric Subepithelial Tumor: Finding Ways to Manage with Shorter Hospitalization Days
}

\author{
Sangjun Lee, M.D. ${ }^{1}$, Sa-Hong Min, M.D. ${ }^{1}$, Ki Bum Park, M.D. ${ }^{2}$, Dong-Wook Kim, M.D. ${ }^{3}$, Yoontaek Lee, M.D. ${ }^{4}$,
} Young Suk Park, M.D. ${ }^{1}$, Sang-Hoon Ahn, M.D. ${ }^{1}$, Do Joong Park, M.D. ', Hyung-Ho Kim, M.D. ${ }^{1,5}$

'Department of Surgery, Seoul National University Bundang Hospital, Seongnam, 2Department of Surgery, Kyungpook National University Hospital, Daegu, ${ }^{3}$ Department of Surgery, Dankook University Hospital, Cheonan, ${ }^{4}$ Department of Surgery, Ehwa University Medical Center, ${ }^{5}$ Department of Surgery, Seoul National University College of Medicine, Seoul, Korea

Purpose: The standard treatment for gastric subepithelial tumor (SET) is surgical resection, which is primarily performed via laparoscopy. The aims of this study were firstly to evaluate factors influencing morbidity and hospitalization after treatment of gastric SET, and secondly, to figure out the factors how to make shorter hospitalization with equal safety.

Methods: We retrospectively enrolled 229 consecutive patients who underwent laparoscopic gastric wedge resection (LGWR) for gastric SET between August 2003 and December 2015. Patients were divided into two groups: the 3 days or less hospitalization group $(\mathrm{N}=82$, group $\mathrm{A})$ and the greater than 3 days hospitalization group ( $\mathrm{N}=147$, group $\mathrm{B})$.

Results: Median tumor size was $3.0 \mathrm{~cm}$ (range, $0.2 \sim 13.0 \mathrm{~cm}$ ) and mean postoperative hospitalization was $4.27 \pm 2.15$ days. There were 6 complications (2.6\%), with no cases of mortality. In group A, tumors were smaller $(3.0 \pm 1.1 \mathrm{~cm}$ vs. $3.6 \pm 1.9 \mathrm{~cm}, p<0.01)$ and more likely to be located on the greater curvature ( $28 \%$ vs. $15 \%, p<0.01)$ compared with group B. The tumor growth pattern (exophytic tumor: $72 \%$ in group A vs. $65 \%$ in group $\mathrm{B}, p=0.25$ ) was not different between the two groups. Multivariate analysis showed that tumor size larger than $5 \mathrm{~cm}$ and posterior wall tumor location were risk factors for longer hospital stay.

Conclusion: We could reduce the hospitalization of patients with gastric SET less than $5 \mathrm{~cm}$ sized and located on other than the posterior wall within 3days. Those patients could be a candidate for day surgery.

Keywords: Laparoscopy, Gastrectomy, Day surgery, Subepithelial
Received April 10, 2019

Revised 1st June 14, 2019

2nd July 29, 2019

Accepted August 16, 2019

Corresponding author

Hyung-Ho Kim

Department of Surgery, Seoul

National University Bundang

Hospital, Gumi-ro 173, 82 Beon-

gil, Bundang-gu, Seongnam 13620,

Korea

Tel: +82-10-3079-7095

Fax: +82-31-787-4055

E-mail: lapakh2@gmail.com

ORCID:

https://orcid.org/0000-0002-8916-0048
This is an Open Access article distributed under the terms of the Creative Commons Attribution Non-Commercial License (http:// creativecommons.org/licenses/by-nc/4.0/) which permits unrestricted non-commercial use, distribution, and reproduction in any medium, provided the original work is properly cited.

\author{
Copyright $@ 2019$ The Journal of Minimally \\ Invasive Surgery. All rights reserved.
}

\section{INTRODUCTION}

Gastrointestinal stromal tumor (GIST) is the most common mesenchymal tumor in the gastrointestinal tract, and in the stomach specifically. Most guidelines recommend that GIST larger than $2 \mathrm{~cm}$ should be surgically resected due to the potential for malignant features. For small GIST, measuring less than $2 \mathrm{~cm}$, the National Comprehensive Cancer Network guideline suggests that tumors showing high risk features should be removed. ${ }^{1}$ Laparoscopic partial gastrectomy (i.e. wedge resection of the stomach) was originally indicated for the resection of tumors less than $2 \mathrm{~cm}$. Recent practice guidelines have recommended the use of laparoscopic resection for GIST smaller than $5 \mathrm{~cm}^{2,3}$ Some investigators reported that 
laparoscopic resection was feasible and safe for tumors up to 8 $\mathrm{cm}$ in diameter. ${ }^{4,5}$ Generally, laparoscopic resection for gastric GIST is considered a technically feasible and safe procedure with better cosmetic outcome and oncologic equivalence to open surgery. ${ }^{2,46}$

As Jia-Qin Cai et al. reported in their meta-analysis and systematic review, laparoscopic gastric wedge resection (LGWR) has a lower complication rate than open surgery. Patients who undergo surgery for gastric SET spend a variable number of in-hospital days for recovery prior to discharge. Certain factors may influence the length of hospital stay, such as tumor characteristics including size, location, and other features. ${ }^{2,8}$

The aim of this study was to evaluate factors influencing morbidity and hospitalization after treatment of gastric SET, in particular, LGWR. Moreover, we aimed to identify factors associated with a shortened hospital stay, and to suggest a criteria with the factors that might allow safe same-day surgery.

\section{MATERIALS AND METHODS}

\section{Patients}

We retrospectively identified 363 cases of gastric SET treated surgically between August 2003 and December 2015 at Seoul National University Bundang Hospital. Among these 363 cases, 229 patients who underwent LGWR for gastric SET were enrolled in this study.

Clinical data were collected from the electronic medical recording system of our hospital and prospectively maintained in a gastric cancer database by our team. Factors investigated included length of hospital stay, age, sex, BMI, ASA score, tumor size, tumor location, tumor growth pattern, complications, and readmission rate after discharge. Tumor size was defined as the largest diameter of the tumor. Postoperative pain was evaluated immediately after transferring patients to wards from operation room, and on postoperative day (POD) \#1 using the Numerical Rating Scale (NRS).

Based on the length of hospital stay, the patients were divided into two groups: group $\mathrm{A}(\mathrm{n}=82)$ included patients hospitalized for 3 days or less postoperatively; group B ( $n=147$ ) included patients hospitalized greater than 3 days.

We statistically evaluated the differences in the analyzed factors between the two groups via a cross-sectional study of the groups.

\section{Statistical analysis}

Analysis of demographic and clinicopathological characteristics was performed using descriptive statistics. Quantitative variables are reported as mean and standard deviation and qualitative variables are reported as frequency and percentage. Comparisons between groups were performed using the chisquare test for qualitative variables and two-sample t-test with normality for quantitative variables. All tests were two-sided and $p$ values less than 0.05 were considered statistically significant. IBM SPSS Statistics ver. 18.0 (IBM Co., Armonk, NY, USA) was used for multivariate analysis.

\section{RESULTS}

\section{Demographic and clinicopathologic characteristics}

Table 1 reports characteristics of patients in groups A and B. In group $\mathrm{A}$, there were 30 males $(36.6 \%)$ and 52 females (63.4\%), with a mean age of $58.3 \pm 12.5$ years and mean body mass index (BMI) of $24.7 \pm 2.82 \mathrm{~kg} / \mathrm{m}^{2}$. Median tumor size was 2.85 $\mathrm{cm}$ (range, 0.6 6.5 cm). An exophytic tumor growth pattern was diagnosed in 59 cases; the remaining 23 cases showed an endophytic growth pattern. Mean number of surgical staplers used was 2.12 \pm 1.07 . Postoperative pain level was $6.72 \pm 1.57$ immediately after surgery, and $3.87 \pm 1.01$ on POD \#1. ASA score, Tumor location is described in Table 1.

In group B, there were 63 males (42.9\%) and 84 females (57.1\%), with a mean age of $57.7 \pm 13.7$ years and mean BMI of $24.3 \pm 3.15 \mathrm{~kg} / \mathrm{m}^{2}$. Median tumor size was $3.0 \mathrm{~cm}$ (range, 0.2 13 $\mathrm{cm})$. An exophytic tumor growth pattern was diagnosed in 95 cases; the remaining 52 cases showed an endophytic growth pattern. The mean number of surgical staplers used was $2.80 \pm$ 1.62. Postoperative pain was $6.26 \pm 1.81$ immediately after surgery and $3.92 \pm 1.49$ on POD \#1.

In group A, tumors were smaller $(3.0 \pm 1.1 \mathrm{~cm}$ vs. $3.6 \pm 1.9$ $\mathrm{cm}, p<0.01$ ). Based on chi-square analysis, there was a significant correlation between tumor location and length of hospitalization $(p=0.01)$. For example, tumors in group A were more frequently located on the greater curvature (GC) $(28.0 \%$ vs. $15.6 \%, p<0.01)$ and less frequently located on the posterior wall (PW) $(14.6 \%$ vs. $36.1 \%, p<0.01)$ (Table 2). Fewer surgical staplers were used in group A $(2.12 \pm 1.07$ vs. $2.79 \pm 1.61$, $p<0.01$ ). There was no significant difference in tumor growth pattern between groups ( $72 \%$ vs. $65 \%, p=0.25$ ). NRS score was not significantly different between groups; $p=0.068$ in immediately after surgery, $p=0.77$ in POD \#1. Pathological biopsy findings showed no significant difference between groups: diagnoses in group A were GIST (67.1\%), schwannoma (17.1\%), and leiomyoma (11.0\%); diagnoses in group B were GIST (63.6\%), schwannoma (8.6\%), and leiomyoma (15.2\%) ( $p=0.17)$.

Multivariate analysis showed that tumor size larger than 5 $\mathrm{cm}$ and tumor location on posterior wall were risk factors for longer hospital stay over 3 days. (Table 3). Concerning tumor 
Table 1. Comparison on the demographic findings, clinicopathologic findings of the patients between group A and group B

\begin{tabular}{|c|c|c|c|}
\hline \multirow{2}{*}{ Variables } & Group $A(H D \leq 3$ days) & Group $B(\mathrm{HD}>3$ days) & \multirow{2}{*}{$p$ value } \\
\hline & $(n=82)$ & $(n=147)$ & \\
\hline Mean Tumor size (cm) & $3.02 \pm 1.08$ & $3.58 \pm 1.88$ & $0.001^{*}$ \\
\hline Age lyears) & $58.3 \pm 12.5$ & $57.7 \pm 13.7$ & 0.746 \\
\hline BMI $\left(\mathrm{kg} / \mathrm{m}^{2}\right)$ & $24.7 \pm 2.82$ & $24.3 \pm 3.15$ & 0.415 \\
\hline No. staples & $2.12 \pm 1.07$ & $2.80 \pm 1.62$ & $0.001^{*}$ \\
\hline Gender & & & 0.353 \\
\hline Male & $30(36.6)$ & $63(42.9)$ & \\
\hline Female & $52(63.4)$ & $84(57.1)$ & \\
\hline ASA score & & & 0.357 \\
\hline | & $35(42.7)$ & $71(48.3)$ & \\
\hline$\|$ & $46(56.1)$ & $71(48.3)$ & \\
\hline III & $1(1.2)$ & $5(3.4)$ & \\
\hline IV & $0(0)$ & $0(0)$ & \\
\hline Pathology & & & 0.167 \\
\hline GIST & $54(65.9)$ & $87(59.2)$ & \\
\hline Schwannoma & $9(11.0)$ & $20(13.6)$ & \\
\hline Leiomyoma & $13(15.9)$ & $12(8.2)$ & \\
\hline Others & $6(7.3)$ & $28(19.0)$ & \\
\hline \multicolumn{4}{|l|}{ Mean NRS score } \\
\hline Immediate postop & $6.26 \pm 1.81$ & $6.72 \pm 1.57$ & 0.068 \\
\hline On POD\#1 & $3.87 \pm 1.01$ & $3.92 \pm 1.49$ & 0.77 \\
\hline Postoperative complication & & & 0.368 \\
\hline No & $82(100)$ & 141 (95.9) & \\
\hline Yes & $0(0)$ & $6(2.6)$ & \\
\hline Fever & $0(0)$ & $1(0.4)$ & \\
\hline Lung & $0(0)$ & $1(0.4)$ & \\
\hline lleus & $0(0)$ & $1(0.4)$ & \\
\hline Bleeding & $0(0)$ & $2(0.9)$ & \\
\hline Perforation & $0(0)$ & $1(0.4)$ & \\
\hline \multicolumn{4}{|c|}{ Re-admission within 30 days after surgery } \\
\hline No & $82(100)$ & 147 (100) & \\
\hline Yes & 0 & 0 & \\
\hline
\end{tabular}

Values are presented as the number $(\%)$ and the mean value \pm standard deviation. $H D=$ hospitalization dayls); BMI=body mass index; GIST=gastrointestinal stromal tumor; $A S A=$ American Society of Anesthesiologists; NRS = numerical rating scales.

size, tumors measuring $5 \mathrm{~cm}$ or larger increased risk of longer hospitalization, or being in group $\mathrm{B}$ (odds ratio $(\mathrm{OR})=4.738$, a 95\% confidence interval $(95 \% \mathrm{CI})$ of $1.544 \sim 14.536(p<0.01))$. Concerning tumor location, tumors located on the posterior wall were significantly associated with longer hospitalization comparing to those on the lesser curvature (LC) (OR 3.197, 95\% CI 1.356 7.538, $p<0.01)$. 
Table 2. Comparison on characteristics of tumors between group A and group $B$

\begin{tabular}{|c|c|c|c|}
\hline \multirow[t]{2}{*}{ Variables } & $\begin{array}{c}\text { Group } A \\
(\mathrm{HD} \leq 3 \text { days })\end{array}$ & $\begin{array}{c}\text { Group B } \\
(\mathrm{HD}>3 \text { days })\end{array}$ & \multirow[t]{2}{*}{$p$ value } \\
\hline & $(n=82)$ & $(n=147)$ & \\
\hline \multicolumn{4}{|l|}{ Tumor recurrence } \\
\hline No & $82(100)$ & 147 (100) & \\
\hline Yes & 0 & 0 & \\
\hline Tumor size $(\mathrm{cm})$ & & & $0.029^{*}$ \\
\hline$\leq 2$ & $11(13.4)$ & $22(15.0)$ & \\
\hline$\geq 2.1$ to $\leq 5$ & $67(81.7)$ & $98(66.7)$ & \\
\hline$\geq 5.1$ to $\leq 10$ & $4(4.9)$ & $24(16.3)$ & \\
\hline$\geq 10.1$ & $0(0)$ & $3(2.0)$ & \\
\hline Tumor location (tubular) & & & 0.116 \\
\hline Upper 1/3 & $38(46.3)$ & $89(60.5)$ & \\
\hline Middle 1/3 & $29(35.4)$ & $38(25.9)$ & \\
\hline Lower $1 / 3$ & $15(18.3)$ & $20(13.6)$ & \\
\hline Tumor location (circular) & & & $0.007^{*}$ \\
\hline Lesser curvature & $21(25.6)$ & 32 (21.8) & \\
\hline Greater curvature & $23(28.0)$ & $23(15.6)$ & \\
\hline Anterior wall & $16(19.5)$ & $27(18.4)$ & \\
\hline Posterior wall & $12(14.6)$ & $53(36.1)$ & \\
\hline Others & $10(12.2)$ & $12(8.1)$ & \\
\hline Tumor growth pattern & & & 0.254 \\
\hline Endophytic & $23(28.0)$ & $52(35.4)$ & \\
\hline exophytic & $59(72.0)$ & $95(64.6)$ & \\
\hline
\end{tabular}

Values are presented as the number $(\%)$. $H D=$ hospitalization day(s).

\section{Complications}

There were 6 (2.6\%) complications, all occurring in group B: 1 case of fever, 1 case of pulmonary complication: atelectasis, 1 case of ileus, 2 cases of bleeding, and 1 case of surgical site perforation. The patient who developed surgical site perforation was treated with re-operation. There were no cases of immediate postoperative mortality. There were no cases of readmission within 30 days after discharge. There was no tumor recurrence in either group.

\section{DISCUSSION}

Reducing hospitalization days can be beneficial for several reasons. First, if a patient is able to be discharged early, medi-
Table 3. Risk factors of longer hospital stay by multivariate analysis

\begin{tabular}{llll}
\multicolumn{1}{c}{ Variables } & \multicolumn{1}{c}{ OR } & \multicolumn{1}{c}{$95 \% \mathrm{Cl}$} & $p$ value \\
\hline Tumor size (vs. $\geq 5 \mathrm{~cm}$ ) & 4.738 & $1.544 \sim 14.536$ & $0.007^{*}$ \\
ASA score & & & 0.447 \\
I to III & 0.8 & $0.076 \sim 8.459$ & 0.853 \\
II to III & 0.557 & $0.053 \sim 5.841$ & 0.626 \\
Circular location & & & $0.009^{*}$ \\
GC to LC & 0.686 & $0.300 \sim 1.564$ & 0.37 \\
AW to LC & 1.284 & $0.548 \sim 3.008$ & 0.566 \\
PW to LC & 3.197 & $1.356 \sim 7.538$ & $0.008^{*}$ \\
Others to LC & 0.826 & $0.293 \sim 2.331$ & 0.718
\end{tabular}

Values are presented as the number. ASA $=$ American Society of Anesthesiologists; $\mathrm{GC}=$ greater curvature; $\mathrm{LC}=$ lesser curvature; $\mathrm{AW}=$ anterior wall; $P W=$ posterior wall.

cal resource consumption by the patient is also reduced., ${ }^{9,10}$ Second, reduced hospital stay is associated with a reduced risk of infection, in particular hospital acquired infection. Hospital acquired infection can be prevented by many factors, including hand hygiene and aseptic technique; reducing the length of the hospital stay itself can also decrease the infection rate. ${ }^{10}$ Third, patients can return to their normal daily routine earlier with more rapid hospital discharge, allowing them to conserve their own resources, such as money and time. ${ }^{9,10}$ Moreover, several studies have found that laparoscopic procedures are associated with a shorter hospital stay compared with open surgery, with similar complication rates. ${ }^{5,70}$ Therefore, we conducted this study to investigate factors influencing the length of hospital stay following LGWR. In the current analysis, mean hospital stay (4.26 days) was similar to that of previous reports.

The most frequent type of SET in our study was GIST (64.8\%). Since GIST has a poor prognosis, complete resection is required in case of symptomatic gastric SET measuring $2 \mathrm{~cm}$ or greater.,111 In the case of GIST, the tumor size itself contributes to tumor T stage. ${ }^{11}$ Because larger gastric SET have a greater chance of being diagnosed as GIST, the treatment of larger SET should be similar to that of GIST. While it is considered safe to treat gastric SET laparoscopically, larger tumors may require higher level surgical skills and carry a greater risk of surgical complications, including bleeding and perforation.

Comparing laparoscopic versus open surgical approaches, many studies have reported no difference in surgical outcomes, and that the laparoscopic approach can be safe and even reduce the length of hospital stay. ${ }^{3-5,9}$ LGWR is associated with reduced pain and fewer wound complications compared 
with open surgery. ${ }^{3,7,110,12}$ In the current study, pain scoring by NRS showed no difference between the two groups, with pain levels improved on POD \#1. The laparoscopic approach allows patients to avoid complications of prolonged general anesthesia. Safety issues with minimally invasive surgery for GIST have been analyzed in previous reports. ${ }^{2}$

In our study, there was no case of re-admission within 30 days after discharge among the total of 229 patients; postoperatively, patients were discharged and followed up in an outpatient clinic. In other words, early discharge did not increase the risk of re-admission.

In multivariate analysis, tumor size and tumor location were the two factors found to significantly increase the risk of prolonged hospitalization. Other factors including laboratory data, age, sex, BMI, or ASA score were not significantly associated with increased hospitalization length.

Tumor growth pattern was also anticipated to affect hospitalization length, but no difference was found between the two groups in the present study. An endophytic pattern was more common among patients with complications. Patients with endophytic SET should be closely observed postoperatively.

For assessing odd ratio with tumor location, lesser curvature was used for reference category. In general, tumors on LC site are difficult to resect because many blood vessels run into stomach and nerves innervate through this site. So there are two difficult points; one is that more delicate surgical procedure is required to clear vessels and nerves; the other is that LC area has higher stricture risk than other areas after resection because less tissue expansion is made than other areas. So, relatively more technical-demanding site or the LC was chosen to assess other area.

With well-designed preoperative indications for patient selection, patients may successfully undergo LGWR with 1 or 2 days of hospitalization, or even on a day surgery basis.

In our hospital, patients with a gastric SET are treated following a protocol of routine care, or a clinical pathway (CP). Our hospital provides this routine care or $\mathrm{CP}$ to patients with small SET, focused on safety and early discharge. If a patient has no significant postoperative complications, he or she will be discharged 2 days after the operation. Following the $\mathrm{CP}$, patients are admitted to the hospital prior to the day of surgery. Preoperative work-up is conducted in an outpatient clinic, and surgery is performed the following day. The patients are maintained on fasting status or NPO at least 6 hours before the operation. On the third day or POD \#1, the patients can eat a semi-fluid diet or a soft blend diet, based on the patient's condition. On the fourth day, the patient is discharged unless there are unstable vital signs, uncontrolled severe pain, or significant complications such as bleeding or perforation.

In our hospital, most gastric SET patients have followed the $\mathrm{CP}$ course. Overall hospitalization days were 5.25 days in 2004, and had been decreased every year by 3.7 days in 2015 (Fig. 1). ${ }^{13}$ There are some reasons of this trend. First, considering our hospital opened in 2003, initial longer hospitalization day may be explained with learning curve. Second, with the similar context of the learning curve, there had been more patients with smaller tumors or earlier stage thanks to the increase of EGD surveillance. So, if there is a more strict criteria about tumor size, tumor location, there might be an influence to decrease hospitalization days.

In our study, the critical pathway process of the hospitalization within 3 days (Group A) was admitted to the hospital 1 day before surgery and dischared one day after surgery. Perhaps reducing the hospitalization for 2 days before and after surgery, which may not be necessary in thoes patinets, we think it would be possible to implement day surgery for those patients.

If a patient shows symptoms or signs for any complication or unstable vital sign, he or she will be administrated for additional one or two days for close monitoring until the problem is resolved. In recent experience of our hospital, patients with routine benign cases tend to have fewer hospitalization days compared to the older days, except for complicated case. In other words, that patients follow the CP course well without extending hospitalization day. Learning curve for the surgical skill, development of surgical tools such as surgical staplers, may contribute to this trend. If a surgeon is well trained enough for laparoscopic gastric SET with well targeted patients who have low risk factors, patients may reduce hospitalization days, even same day surgery.

In order to provide routine, safe care, steps should be taken to avoid complications. In the current study, the overall com-

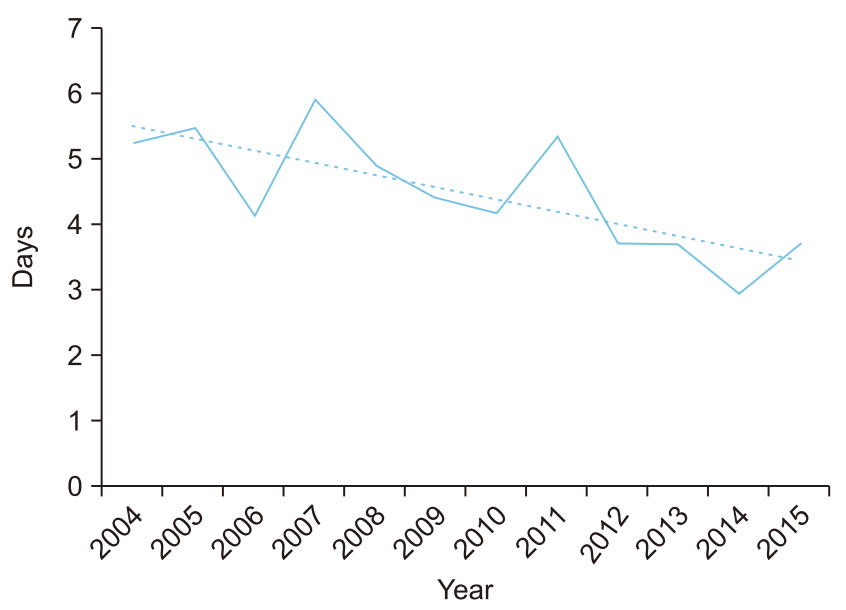

Fig. 1. Trend of overall average hospitalization days of the patients treated with gastric SET. 
plication rate was 2.6\%; this is relatively low in comparison to a previous study, which previously reported a $10 \%$ complication rate. ${ }^{14}$ In most cases, the postoperative course was generally uneventful with the exception of six patients; no case of mortality occurred. One patient with surgical site perforation and one patient with bleeding required re-operation; after re-operation, the patients were able to be discharged without further complications. The remaining five patients were conservatively managed without the need for additional interventions. While rare, a variety of complications can occur postoperatively. Mild complications such as fever, atelectasis, and ileus are not uncommon in surgeries requiring general anesthesia, thus routine postoperative care may reduce their occurrence. Severe complications such as bleeding and perforation, however, should be evaluated more cautiously because they could lead to major morbidity or fatality. These complications may occur when resecting the tumor with a surgical stapler.

In the current study, patients who developed bleeding complications presented with decreased serum hemoglobin and increased heart rate. The patient who developed perforation showed symptoms on physical exam including severe abdominal pain, tenderness, and rebound tenderness after starting a diet. With skillful manipulation such as a tension free stapling maneuver, meticulous bleeding control, identification and repair of wall defects, and close postoperative physical examination, these complications may be prevented.

Our study has some limitations. Data were retrospectively reviewed, and the study was conducted at a single center. Concerning tumor recurrence, no recurrence occurred during follow-up in our patient population. However, follow-up at the outpatient clinic was not consistent for all patients. Further study with a longer follow-up may be required to investigate the recurrence rate.

\section{CONCLUSION}

Smaller tumor size and tumor location on other than PW were more common among patients with a shorter hospital stay. We could reduce the hospitalization of patients with gastric SET less than $5 \mathrm{~cm}$ sized and located on other than the posterior wall within 3 days. Those patients could be a candidate for day surgery.

\section{ORCID}

Sangjun Lee, https://orcid.org/0000-0001-9498-0254

Sa-Hong Min, https://orcid.org/0000-0002-6150-7935

Ki Bum Park, https://orcid.org/0000-0001-5404-5667

Dong-Wook Kim, https://orcid.org/0000-0002-3552-5988
Yoontaek Lee, https://orcid.org/0000-0003-2643-2007

Young Suk Park, https://orcid.org/0000-0002-6352-9759

Sang-Hoon Ahn, https://orcid.org/0000-0001-8827-3625

Do Joong Park, https://orcid.org/0000-0001-9644-6127

Hyung-Ho Kim, https://orcid.org/0000-0002-8916-0048

\section{AUTHORS' CONTRIBUTIONS}

Conceptualization: SJL, YTL, HHK. Formal analysis: SJL, DWK. Methodology: SJL, KBP. Writing-original draft: SJL. Writing-review and editing: SJL, SHM, YSP, SHA, DJP, HHK.

\section{CONFLICT OF INTEREST}

None.

\section{FUNDING}

None.

\section{ACKNOWLEDGMENTS}

None.

\section{REFERENCES}

1) Yang HK, Park DJ, Lee HJ, Kim HH, Kim WH, Lee KU. Clinicopathologic characteristics of gastrointestinal stromal tumor of the stomach. Hepatogastroenterology 2008:55:1925-1930.

2) Yin $X$, Yin $Y$, Chen H, et al. Comparison Analysis of Three Different Types of Minimally Invasive Procedures for Gastrointestinal Stromal Tumors $</=5 \mathrm{~cm}$. J Laparoendosc Adv Surg Tech A 2018; 28:58-64.

3) Lin J, Huang $\mathrm{C}$, Zheng $\mathrm{C}$, et al. Laparoscopic versus open gastric resection for larger than $5 \mathrm{~cm}$ primary gastric gastrointestinal stromal tumors (GIST): a size-matched comparison. Surg Endosc 2014;28:2577-2583.

4) Kim KH, Kim MC, Jung GJ, Kim SJ, Jang JS, Kwon HC. Long term survival results for gastric GIST: is laparoscopic surgery for large gastric GIST feasible? World J Surg Oncol 2012;10:230.

5) Karakousis GC, Singer S, Zheng J, et al. Laparoscopic versus open gastric resections for primary gastrointestinal stromal tumors (GISTs): a size-matched comparison. Ann Surg Oncol 2011;18: 1599-1605.

6) Samardzic J, Hreckovski B, Hasukic I, Hasukic S. Laparoscopic Wedge Resection of Gastric Stromal Tumor (GIST). Med Arch 2015;69:203-205.

7) Cai JQ, Chen K, Mou YP, et al. Laparoscopic versus open wedge resection for gastrointestinal stromal tumors of the stomach: a single-center 8-year retrospective cohort study of 156 patients 
with long-term follow-up. BMC Surg 2015;15:58.

8) Nguyen SQ, Divino CM, Wang JL, Dikman SH. Laparoscopic management of gastrointestinal stromal tumors. Surg Endosc 2006; 20:713-716.

9) Choi CI, M.d, Kim DH, et al. The Comparison between Laparoscopic Wedge Resection and Open Wedge Resection for Gastric Gastrointestinal Stromal Tumors. J Minim Invasive Surg 2010;13: 6-10.

10) de'Angelis N, Brunetti F, Felli E, et al. Laparoscopic versus open gastric wedge resection for primary gastrointestinal tumors: clinical outcomes and health care costs analysis. Surg Laparosc Endosc Percutan Tech 2015;25:143-146.

11) Nishida $T$, Hirota S, Yanagisawa A, et al. Clinical practice guide- lines for gastrointestinal stromal tumor (GIST) in Japan: English version. Int J Clin Oncol 2008;13:416-430.

12) Liao GQ, Chen T, Qi XL, et al. Laparoscopic management of gastric gastrointestinal stromal tumors: A retrospective 10-year single-center experience. World J Gastroenterol 2017;23:35223529.

13) Hopper AN, Jamison MH, Lewis WG. Learning curves in surgical practice. Postgrad Med J 2007;83:777-779.

14) Matsuhashi N, Osada S, Yamaguchi K, et al. Long-term outcomes of treatment of gastric gastrointestinal stromal tumor by laparoscopic surgery: review of the literature and our experience. Hepatogastroenterology 2013;60:2011-2015. 